\title{
DERIVATION OF THE DOUBLE POROSITY MODEL OF SINGLE PHASE FLOW VIA HOMOGENIZATION THEORY*
}

\author{
TODD ARBOGAST $\dagger$, JIM DOUGLAS, JR.†, AND ULRICH HORNUNG $\ddagger$
}

\begin{abstract}
A general form of the double porosity model of single phase flow in a naturally fractured reservoir is derived from homogenization theory. The microscopic model consists of the usual equations describing Darcy flow in a reservoir, except that the porosity and permeability coefficients are highly discontinuous. Over the matrix domain, the coefficients are scaled by a parameter $\epsilon$ representing the size of the matrix blocks. This scaling preserves the physics of the flow in the matrix as $\epsilon$ tends to zero. An effective macroscopic limit model is obtained that includes the usual Darcy equations in the matrix blocks and a similar equation for the fracture system that contains a term representing a source of fluid from the matrix. The convergence is shown by extracting weak limits in appropriate Hilbert spaces. A dilation operator is utilized to see the otherwise vanishing physics in the matrix blocks as $\epsilon$ tends to zero.
\end{abstract}

Key words. porous medium, double porosity, fractured reservoir, homogenization

AMS(MOS) subject classifications. $76 \mathrm{~S} 05$

1. Introduction. It has long been known that the porous rock that composes a petroleum reservoir may contain many cracks or fractures. A naturally fractured reservoir is one that has throughout its extent many interconnected fracture planes. For over 30 years it has been known that flow in such reservoirs is not like that in unfractured reservoirs [16]. Instead, the flow acts as if the reservoir possessed two porous structures, one associated to the system of fractures, and the other associated to the porous rock (the matrix). This double porosity/permeability concept has been used to model the flow of a single component in a single phase within a naturally fractured reservoir since around 1960 [5], [14], [18], [21].

More recently, a general form of the double porosity/permeability model has been described [3], [10]. The earlier models can be considered as approximations to this more general model [3]. It was derived on physical grounds under the main assumption that the fluid pressure (or, equivalently, density) is uniform at the surface of each matrix block. Herein we will derive this general model from the point of view of homogenization theory [6], [17]. It will be seen that the model is in some sense the limit of a family of microscopic models in which the sizes of the matrix blocks tend to zero (hence, the fluid indeed becomes uniform at the surfaces of the blocks).

A straightforward homogenization of the entire reservoir would yield a single porosity model with some average permeability [1], [7]. This would be quite inadequate since two very distinct porous structures are present in the reservoir and their interaction has a strong influence on the flow characteristics. This interaction is a fine structure process whose effect only must be homogenized; the process itself must be retained on a microscopic level.

\footnotetext{
* Received by the editors January 16, 1989; accepted for publication August 1, 1989. This work was supported in part by the National Science Foundation.

$\dagger$ Department of Mathematics, Purdue University, West Lafayette, Indiana 47907. Germany.

$\ddagger$ Scientific Hornung Institute (SCHI), P.O. Box 1222, D-8014 Neubiberg, Federal Republic of
} 
We will use a variant of the homogenization technique that was used by Hornung and Jäger to describe catalytic reactions in a porous medium [12]. There, the driving mechanism was the chemical process of catalytic reactions that takes place on a microscopic scale. The overall (or homogenized) behavior of the system could be obtained only when a careful modeling of these microscopic reactions was maintained as the medium was homogenized. See also [11] for a similar situation involving displacement in mobile and immobile water and [20] for an example from chromatography. This technique is also discussed in a formal sense in a related paper where Arbogast, Douglas, and Hornung consider two-component flows in naturally fractured reservoirs [4]; see also [9]. An independent study of diffusion problems in fractured porous media obtained by homogenization is given by Hornung and Showalter [13].

The remainder of the paper is as follows. In the next section, we will write down the equations that describe the microscopic nature of the flow in a naturally fractured reservoir. We will scale the equations by a parameter $\epsilon$, where $\epsilon$ is the size of the matrix blocks. This places the model in a series of problems from which, as $\epsilon$ tends to zero, we obtain our homogenized macroscopic model, presented in $\S 3$. In the final section, we prove that the solutions of the microscopic model converge weakly to those of the macroscopic model in appropriate Hilbert spaces and describe some of the mathematical properties of the limit model.

2. The microscopic model. We consider the reservoir $\Omega \subset \mathbb{R}^{3}$ to be a bounded, two-connected domain with a periodic structure. More precisely, $\Omega$ is a union of disjoint parallelepiped cell domains congruent to a standard one $\mathcal{Q}$ :

$$
\bar{\Omega}=\bigcup_{c \in \mathcal{A}}(\overline{\mathcal{Q}}+c), \quad\left(\mathcal{Q}+c_{1}\right) \cap\left(\mathcal{Q}+c_{2}\right)=\emptyset \quad \text { whenever } c_{1} \neq c_{2} \in \mathcal{A},
$$

where $\mathcal{A}$ is an appropriate finite lattice of translations containing the origin and the overline denotes closure (see Fig. 1). The cell $\mathcal{Q}$ can be decomposed into three pieces, a compactly contained, two-connected domain $\mathcal{Q}_{m}$ representing the matrix block part, the surrounding connected fracture domain $\mathcal{Q}_{f}$, and a smooth internal boundary piece $\partial \mathcal{Q}_{m}$ (see Fig. 2).

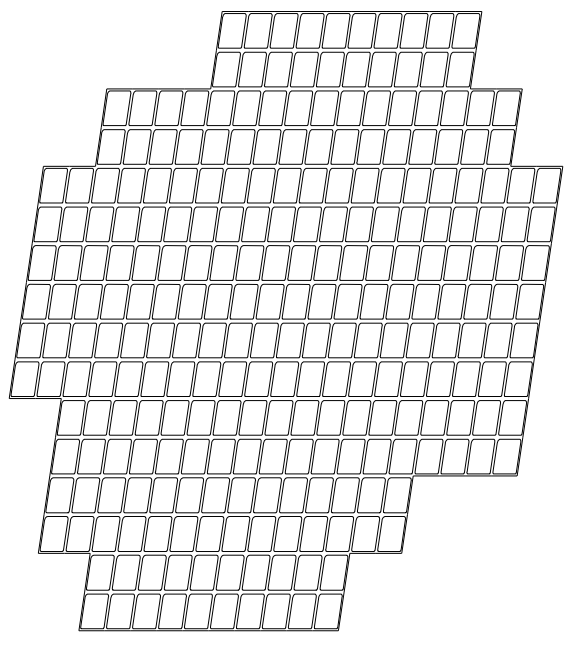

$\Omega$

Fig. 1. The reservoir $W$, depicting its periodic structure. 


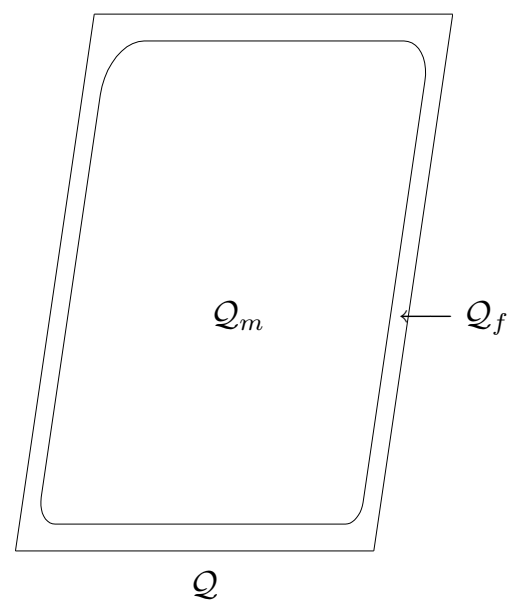

Fig. 2. The standard cell $Q$.

To homogenize the reservoir, we will let tend to zero the (linear) size $\epsilon$ of the cells ( $\epsilon=1$ in the microscopic model). Extend $\mathcal{A}$ to an infinite lattice $\mathcal{A}^{\prime}$. For $\epsilon>0$, let the fracture and matrix domains be denoted, respectively, by

$$
\Omega_{f}^{\epsilon}=\Omega \cap \bigcup_{c \in \mathcal{A}^{\prime}} \epsilon\left(\mathcal{Q}_{f}+c\right) \quad \text { and } \quad \Omega_{m}^{\epsilon}=\Omega \cap \bigcup_{c \in \mathcal{A}^{\prime}} \epsilon\left(\mathcal{Q}_{m}+c\right)
$$

To avoid unimportant technicalities relating to the boundary of $\Omega$, assume that the $\epsilon$ 's form a sequence for which $\partial \Omega \subset \partial \Omega_{f}^{\epsilon}$.

Let us define some notation and make some physical assumptions before setting up the microscopic model. Denote by $\rho^{\epsilon}(x, t)$ and $\sigma^{\epsilon}(x, t)$ the density of the fluid in $\Omega_{f}^{\epsilon}$ and $\Omega_{m}^{\epsilon}$, respectively. Assume that the fluid is a liquid of viscosity $\mu$ and constant compressibility $c$; that is, the pressure $p$ and the density satisfy the equations of state:

$$
d \rho=c \rho d p \quad \text { and } \quad d \sigma=c \sigma d p .
$$

Of course we will assume that the fluid flows according to Darcy's law in the matrix $\Omega_{m}^{\epsilon}$, where we will let $k^{\epsilon}(x)=k(x / \epsilon)$ and $\phi^{\epsilon}(x)=\phi(x / \epsilon)$ denote the porosity and (possibly tensor) permeability, respectively. These quantities should be periodic of period $\mathcal{Q}$ (reflecting the periodicity of the matrix blocks over $\Omega$-more generally we could assume that the fixed properties of the matrix are of the form $\psi^{\epsilon}(x)=\psi(x, x / \epsilon)$, varying over the reservoir in the first argument and periodic in the second).

We will also assume Darcy's law is valid in the fractures $\Omega_{f}^{\epsilon}$. Clearly this is not strictly correct; however, this has been done in the petroleum engineering literature [14], [18], evidently by considering the fractures to be partially filled with rock debris. In any case, Darcy's law should hold as $\epsilon$ tends to zero [3], [5], [16], [19], [21]; our main interest lies in determining the correct form of the interaction between the matrix and fracture systems. So, let $\Phi^{*}(x)(\approx 1)$ and $K^{*}(x)$ (very large) denote the porosity and scalar permeability of the fracture domain, extended over all of $\Omega$. These four quantities are uniformly positive ( $k$ being symmetric, uniformly positive-definite), and each is assumed smooth and bounded.

Finally, choose some smooth and bounded reference density functions $\rho_{\text {ref }}(x)$ and $\sigma_{\text {ref }}^{\epsilon}(x)=\sigma_{\text {ref }}(x / \epsilon)$, where $\sigma_{\text {ref }}(x)$ is periodic as above. To linearize the equations, we 
will approximate the effects of gravity as follows:

$$
\left(\rho^{\epsilon}\right)^{2} \approx \rho_{\text {ref }}\left(2 \rho^{\epsilon}-\rho_{\text {ref }}\right), \quad\left(\sigma^{\epsilon}\right)^{2} \approx \sigma_{\text {ref }}^{\epsilon}\left(2 \sigma^{\epsilon}-\sigma_{\text {ref }}^{\epsilon}\right) .
$$

The flow in the two domains is then described by conservation of mass combined with Darcy's law. Using our assumptions, in the fracture domain we have

$$
\begin{aligned}
& \Phi^{*} \rho_{t}^{\epsilon}-\nabla \cdot\left\{\frac{K^{*}}{\mu c}\left[\nabla \rho^{\epsilon}+c g \rho_{\mathrm{ref}}\left(2 \rho^{\epsilon}-\rho_{\mathrm{ref}}\right)\right]\right\}=f \quad \text { for } x \in \Omega_{f}^{\epsilon}, \quad t>0, \\
& \left\{\frac{K^{*}}{\mu c}\left[\nabla \rho^{\epsilon}+c g \rho_{\mathrm{ref}}\left(2 \rho^{\epsilon}-\rho_{\mathrm{ref}}\right)\right]\right\} \cdot \nu \\
& \quad=\epsilon\left\{\frac{k^{\epsilon}}{\mu c}\left[\epsilon \nabla \sigma^{\epsilon}+c g \sigma_{\mathrm{ref}}^{\epsilon}\left(2 \sigma^{\epsilon}-\sigma_{\mathrm{ref}}^{\epsilon}\right)\right]\right\} \cdot \nu \quad \text { for } x \in \partial \Omega_{m}^{\epsilon}, \quad t>0, \\
& \rho^{\epsilon}=\rho_{\text {init }} \quad \text { for } x \in \Omega_{f}^{\epsilon}, \quad t=0
\end{aligned}
$$

here, the subscript $t$ denotes partial differentiation in time, $g$ is the gravitational constant vector, $f(x, t)$ represents external sources/sinks, $\nu(x)$ is the outer unit normal (to $\partial \Omega_{m}^{\epsilon}$, in this case), and $\rho_{\text {init }}(x)$ is the specified initial density.

Similarly in the matrix domain,

$$
\begin{aligned}
& \phi^{\epsilon} \sigma_{t}^{\epsilon}-\epsilon \nabla \cdot\left\{\frac{k^{\epsilon}}{\mu c}\left[\epsilon \nabla \sigma^{\epsilon}+c g \sigma_{\text {ref }}^{\epsilon}\left(2 \sigma^{\epsilon}-\sigma_{\text {ref }}^{\epsilon}\right)\right]\right\}=f \text { for } x \in \Omega_{m}^{\epsilon}, \quad t>0, \\
& \sigma^{\epsilon}=\rho^{\epsilon} \quad \text { for } x \in \partial \Omega_{m}^{\epsilon}, \quad t>0 \\
& \sigma^{\epsilon}=\rho_{\text {init }} \quad \text { for } x \in \Omega_{m}^{\epsilon}, \quad t=0 .
\end{aligned}
$$

The two boundary conditions (2.2) and (2.5) represent conservation of mass flux and continuity of pressure, respectively, between the two domains. We should also assume a no-flow Neumann condition on the fracture density on $\partial \Omega \subset \partial \Omega_{f}^{\epsilon}$ :

$$
\left\{\frac{K^{*}}{\mu c}\left[\nabla \rho^{\epsilon}+c g \rho_{\mathrm{ref}}\left(2 \rho^{\epsilon}-\rho_{\mathrm{ref}}\right)\right]\right\} \cdot \nu=0 \quad \text { for } x \in \partial \Omega, \quad t>0 .
$$

We should make some remarks on the $\epsilon$ scaling factors. They can be viewed as coming from a dimensional analysis of the equations for an individual matrix block; they provide the correct scaling for the flow as the block size shrinks. That is, the form of the matrix equations is preserved on the standard cell independently of the value of $\epsilon$, thereby giving a double porosity model in the limit. This will be seen explicitly in $§ 4$. Alternatively, we may say that the flow between the matrix and fractures is conserved in some sense, as it is prevented from degenerating or blowing up as $\epsilon \rightarrow 0$ [4]. Essentially, the matrix permeability has been scaled by $\epsilon^{2}$, whereas the gravitational term has been compensated by $\epsilon^{-1}$. We might instead decide not to compensate gravity, in which case the macroscopic model would have no gravitational terms in the matrix equations.

3. The macroscopic model. We will show in the next section that, as the scaling parameter $\epsilon$ tends to zero, the microscopic model converges in some sense to the model described below.

We must first define some symbols. Define the macroscopic fracture porosity as

$$
\Phi(x)=\frac{\left|\mathcal{Q}_{f}\right|}{|\mathcal{Q}|} \Phi^{*}(x)
$$


where $|\cdot|$ denotes the volume of the set. Let us define the auxiliary functions $\omega_{j}(y)$, $j=1,2,3$, periodic of period $\mathcal{Q}$, as the solutions modulo constants of

$$
\begin{aligned}
& \Delta \omega_{j}=0 \quad \text { for } y \in \mathcal{Q}_{f}, \\
& \nabla \omega_{j} \cdot \nu=-e_{j} \cdot \nu=-\nu_{j} \quad \text { for } y \in \partial \mathcal{Q}_{m},
\end{aligned}
$$

where $e_{j}$ is the unit vector in the $j$ th direction. Now we can define the effective macroscopic fracture system permeability tensor $K$ componentwise as

$$
K_{i j}(x)=\frac{1}{|\mathcal{Q}|} K^{*}(x)\left\{\left|\mathcal{Q}_{f}\right| \delta_{i j}+\int_{\mathcal{Q}_{f}} \partial_{i} \omega_{j} d y\right\},
$$

where $\delta_{i j}$ is the Kronecker symbol and $\partial_{i}=\partial / \partial y_{i}$.

With $f_{m}$ being defined in (3.11) below, the macroscopic fracture density $\rho(x, t)$ satisfies

$$
\begin{aligned}
& \Phi \rho_{t}-\nabla \cdot\left\{\frac{K}{\mu c}\left[\nabla \rho+c g \rho_{\mathrm{ref}}\left(2 \rho-\rho_{\mathrm{ref}}\right)\right]\right\}=f+f_{m} \quad \text { for } x \in \Omega, \quad t>0, \\
& \left\{\frac{K}{\mu c}\left[\nabla \rho+c g \rho_{\mathrm{ref}}\left(2 \rho-\rho_{\mathrm{ref}}\right)\right]\right\} \cdot \nu=0 \quad \text { for } x \in \partial \Omega, \quad t>0, \\
& \rho=\rho_{\text {init }} \quad \text { for } x \in \Omega, \quad t=0 .
\end{aligned}
$$

As $\epsilon$ tends to zero, we obtain an infinite number of matrix blocks, one for each $x$. Hence, for each $x \in \Omega$, we have a matrix density function $\sigma(x, y, t)$, which is determined from

$$
\begin{aligned}
& \phi(y) \sigma_{t}-\nabla_{y} \cdot\left\{\frac{k(y)}{\mu c}\left[\nabla_{y} \sigma+c g \sigma_{\mathrm{ref}}(y)\left(2 \sigma-\sigma_{\mathrm{ref}}(y)\right)\right]\right\}=f(x, t) \\
& \sigma=\rho(x, t) \quad \text { for } y \in \partial \mathcal{Q}_{m}, \quad t>0, \\
& \sigma=\rho_{\text {init }}(x) \quad \text { for } y \in \mathcal{Q}_{m}, \quad t=0,
\end{aligned}
$$

where $\nabla_{y}$ is the gradient with respect to the $y$ variable. Finally, the matrix source term $f_{m}$ is defined by

$$
f_{m}(x, t)=-\frac{1}{|\mathcal{Q}|} \int_{\mathcal{Q}_{m}} \phi(y) \sigma_{t}(x, y, t) d y
$$

Except for some minor differences, this is exactly the general model originally described in [3] and [10]. Two obvious but unimportant differences are that in the original formulation of the model, the matrix equations do not contain either gravity or external sources; these terms could easily be included. In any case, they are of little mathematical consequence. The interesting differences are associated to the homogenization process itself. The original formulation (and, in fact, the actual problem) had a finite number of finite sized blocks; whereas in the homogenized version of the model, there is a continuum of blocks having no size, since $\epsilon$ tended to zero. This gives rise to subtle differences in the form of the matrix boundary and initial conditions (3.9) and (3.10) as well as a difference in the definition of the matrix source term (3.11). However, there is no difference of any real substance. The boundary and initial conditions of the matrix problems of both formulations are constant with respect to the space variables of the block. Of course in its original form, some representative 
value of the fracture density over the finite-sized block needed to be used. The matrix source terms of both formulations represent the total average flow out of the matrix blocks; this flow needed to be placed explicitly over the entire extent of the finite-sized block in the original version. In practice, the two formulations of the model would be discretized in essentially the same way, since we would need to restrict to a finite number of matrix block problems.

4. The convergence of the homogenization process. We begin by defining some notation. Denote the Sobolev space of functions with derivatives of order $m$ in $L^{p}$ by $W^{m, p}$, and set $H^{m}=W^{m, 2}$. Let $H_{0}^{1}$ denote the closure in $H^{1}$ of $C_{0}^{\infty}$, the infinitely differentiable functions with compact support. Denote by $W^{m, p}\left(W^{n, q}\right)$ the space of $W^{n, q}$-valued functions in $W^{m, p}$. Let $J=(0, T], T>0$, be the time interval of interest. We will make use of several bilinear integration forms. Denote integration in space over the domain $*$ by $(\cdot, \cdot)_{*}$, where $*$ is some subset of $\Omega, \mathcal{Q}$, or $\Omega \times \mathcal{Q}$, and where $*$ determines which of the variables to integrate over. Also, denote integration in space over $*$ and in time over $J$ by $\langle\cdot, \cdot\rangle_{*}$. Let us simplify the notation a bit by setting

$$
\begin{gathered}
\Lambda(x)=\frac{K^{*}(x)}{\mu c}, \quad \lambda^{\epsilon}(x)=\frac{k^{\epsilon}(x)}{\mu c}, \quad \lambda(y)=\frac{k(y)}{\mu c}, \\
\Gamma(x)=c g \rho_{\mathrm{ref}}(x), \quad \gamma^{\epsilon}(x)=c g \sigma_{\mathrm{ref}}^{\epsilon}(x), \quad \gamma(y)=c g \sigma_{\mathrm{ref}}(y) .
\end{gathered}
$$

Finally, let $\chi_{f}^{\epsilon}$ denote the characteristic function of $\Omega_{f}^{\epsilon}$.

Assume throughout that $f \in L^{2}\left(J ; L^{2}(\Omega)\right), \rho_{\text {init }} \in H^{1}(\Omega), \rho_{\text {ref }} \in W^{1, \infty}(\Omega)$, and $\sigma_{\text {ref }} \in W^{1, \infty}\left(\mathcal{Q}_{m}\right)$. The latter two conditions can be relaxed somewhat (see [3]).

TheOREM 1. For each $\epsilon$, there exists a unique solution to the microscopic model, and $\rho^{\epsilon} \in H^{1}\left(J ; L^{2}\left(\Omega_{f}^{\epsilon}\right)\right) \cap L^{\infty}\left(J ; H^{1}\left(\Omega_{f}^{\epsilon}\right)\right)$ and $\sigma^{\epsilon} \in H^{1}\left(J ; L^{2}\left(\Omega_{m}^{\epsilon}\right)\right) \cap L^{\infty}\left(J ; H^{1}\left(\Omega_{m}^{\epsilon}\right)\right)$.

Proof. Recall that $\nu$ is the outer unit normal. Then for $\varphi \in H^{1}(\Omega)$,

$$
-\int_{\partial \Omega_{f}^{\epsilon}} \Lambda(x)\left[\nabla \rho^{\epsilon}(x, t)+\Gamma(x)\left(2 \rho^{\epsilon}(x, t)-\rho_{\mathrm{ref}}(x)\right)\right] \cdot \nu \varphi(x) d s(x)
$$

$$
\begin{aligned}
& =\int_{\partial \Omega_{m}^{\epsilon}} \epsilon \lambda^{\epsilon}(x)\left[\epsilon \nabla \sigma^{\epsilon}(x, t)+\gamma^{\epsilon}(x)\left(2 \sigma^{\epsilon}(x, t)-\sigma_{\mathrm{ref}}^{\epsilon}(x)\right)\right] \cdot \nu \varphi(x) d s(x) \\
& =\left(\epsilon \lambda^{\epsilon}\left[\epsilon \nabla \sigma^{\epsilon}+\gamma^{\epsilon}\left(2 \sigma^{\epsilon}-\sigma_{\mathrm{ref}}^{\epsilon}\right)\right], \nabla \varphi\right)_{\Omega_{m}^{\epsilon}}+\left(\epsilon \nabla \cdot \lambda^{\epsilon}\left[\epsilon \nabla \sigma^{\epsilon}+\gamma^{\epsilon}\left(2 \sigma^{\epsilon}-\sigma_{\mathrm{ref}}^{\epsilon}\right)\right], \varphi\right)_{\Omega_{m}^{\epsilon}} \\
& =\left(\epsilon \lambda^{\epsilon}\left[\epsilon \nabla \sigma^{\epsilon}+\gamma^{\epsilon}\left(2 \sigma^{\epsilon}-\sigma_{\mathrm{ref}}^{\epsilon}\right)\right], \nabla \varphi\right)_{\Omega_{m}^{\epsilon}}+\left(\phi^{\epsilon} \sigma_{t}^{\epsilon}, \varphi\right)_{\Omega_{m}^{\epsilon}}-(f, \varphi)_{\Omega_{m}^{\epsilon}} .
\end{aligned}
$$

Hence, in weak form the microscopic model is

$$
\begin{aligned}
& \left(\Phi^{*} \rho_{t}^{\epsilon}, \varphi\right)_{\Omega_{f}^{\epsilon}}+\left(\Lambda\left[\nabla \rho^{\epsilon}+\Gamma\left(2 \rho^{\epsilon}-\rho_{\mathrm{ref}}\right)\right], \nabla \varphi\right)_{\Omega_{f}^{\epsilon}}+\left(\phi^{\epsilon} \sigma_{t}^{\epsilon}, \varphi\right)_{\Omega_{m}^{\epsilon}} \\
& \quad+\left(\epsilon \lambda^{\epsilon}\left[\epsilon \nabla \sigma^{\epsilon}+\gamma^{\epsilon}\left(2 \sigma^{\epsilon}-\sigma_{\mathrm{ref}}^{\epsilon}\right)\right], \nabla \varphi\right)_{\Omega_{m}^{\epsilon}}=(f, \varphi)_{\Omega}, \quad \varphi \in H^{1}(\Omega), \\
& \quad\left(\phi^{\epsilon} \sigma_{t}^{\epsilon}, \psi\right)_{\Omega_{m}^{\epsilon}}+\left(\epsilon \lambda^{\epsilon}\left[\epsilon \nabla \sigma^{\epsilon}+\gamma^{\epsilon}\left(2 \sigma^{\epsilon}-\sigma_{\mathrm{ref}}^{\epsilon}\right)\right], \nabla \psi\right)_{\Omega_{m}^{\epsilon}} \\
& \quad=(f, \psi)_{\Omega_{m}^{\epsilon}}, \quad \psi \in H_{0}^{1}\left(\Omega_{m}^{\epsilon}\right), \\
& \sigma^{\epsilon}=\rho^{\epsilon} \quad \text { for } x \in \partial \Omega_{m}^{\epsilon}, \quad t>0 .
\end{aligned}
$$


Note that if we let

$$
\theta^{\epsilon}= \begin{cases}\rho^{\epsilon} & \text { for } x \in \Omega_{f}^{\epsilon}, \\ \sigma^{\epsilon} & \text { for } x \in \Omega_{m}^{\epsilon},\end{cases}
$$

then (4.2) is a weak form of

$$
\begin{aligned}
& \alpha^{\epsilon} \theta_{t}^{\epsilon}-\nabla \cdot \kappa^{\epsilon}\left[\nabla \theta^{\epsilon}+\beta^{\epsilon}\left(2 \theta^{\epsilon}-\theta_{\text {ref }}^{\epsilon}\right)\right]=f \quad \text { for } x \in \Omega, \quad t>0, \\
& \kappa^{\epsilon}\left[\nabla \theta^{\epsilon}+\beta^{\epsilon}\left(2 \theta^{\epsilon}-\theta_{\text {ref }}^{\epsilon}\right)\right] \cdot \nu=0 \quad \text { for } x \in \partial \Omega, \quad t>0, \\
& \theta^{\epsilon}=\rho_{\text {init }} \quad \text { for } x \in \Omega, \quad t=0,
\end{aligned}
$$

where

$$
\begin{array}{cl}
\alpha^{\epsilon}=\chi_{f}^{\epsilon} \Phi^{*}+\left(1-\chi_{f}^{\epsilon}\right) \phi^{\epsilon}, & \kappa^{\epsilon}=\chi_{f}^{\epsilon} \Lambda+\left(1-\chi_{f}^{\epsilon}\right) \epsilon^{2} \lambda^{\epsilon}, \\
\beta^{\epsilon}=\chi_{f}^{\epsilon} \Gamma+\left(1-\chi_{f}^{\epsilon}\right) \epsilon^{-1} \gamma^{\epsilon}, & \theta_{\mathrm{ref}}^{\epsilon}=\chi_{f}^{\epsilon} \rho_{\mathrm{ref}}+\left(1-\chi_{f}^{\epsilon}\right) \sigma_{\mathrm{ref}}^{\epsilon} .
\end{array}
$$

This is a single well-posed parabolic problem (with discontinuous coefficients). It is known (and easily shown from the a priori estimates of Lemma 1 below) that there exists a unique solution in $H^{1}\left(J ; L^{2}(\Omega)\right) \cap L^{\infty}\left(J ; H^{1}(\Omega)\right)$. By restriction, we obtain $\rho^{\epsilon}$ and $\sigma^{\epsilon}$ as required.

For each $\epsilon$, we will define a dilation operator " $~$ " taking measurable functions on $\Omega_{r}^{\epsilon}, r=f, m$, or blank, to measurable functions on $\Omega \times \mathcal{Q}_{r}$. First let $c^{\epsilon}(x)$ denote the lattice translation point of the $\epsilon$-cell domain containing $x$; that is, $c^{\epsilon}: \Omega \rightarrow \epsilon \mathcal{A}^{\prime}$ is such that $x \in \epsilon \mathcal{Q}+c^{\epsilon}(x)$. Since the cells are disjoint and fill up space (after dividing up the boundaries of the cells in some nonoverlapping way), $c^{\epsilon}$ is well defined. Then we can define

$$
\tilde{\psi}(x, y)=\psi\left(\epsilon y+c^{\epsilon}(x)\right),
$$

where the $\epsilon$ (and the $r$ ) is implicit. We can now state our main result.

THEOREM 2. The solution $\left(\rho^{\epsilon}, \sigma^{\epsilon}\right)$ of the microscopic model converges as $\epsilon \rightarrow 0$ to the unique solution $(\rho, \sigma)$ of the macroscopic model in the following sense:

$$
\begin{aligned}
\chi_{f}^{\epsilon} \Phi^{*} \rho^{\epsilon} & \longrightarrow \Phi \rho \text { in } H^{1}\left(J ; L^{2}(\Omega)\right) \text { weakly, } \\
\chi_{f}^{\epsilon} \Lambda\left[\nabla \rho^{\epsilon}+\Gamma\left(2 \rho^{\epsilon}-\rho_{\mathrm{ref}}\right)\right] & \longrightarrow \frac{K}{\mu c}\left[\nabla \rho+\Gamma\left(2 \rho-\rho_{\mathrm{ref}}\right)\right] \quad \text { in } L^{2}\left(J ; L^{2}(\Omega)\right) \text { weakly, } \\
\tilde{\sigma}^{\epsilon} & \longrightarrow \sigma \quad \text { in } L^{2}\left(\Omega ; H^{1}\left(\mathcal{Q}_{m} \times J\right)\right) \text { weakly. }
\end{aligned}
$$

The proof will be accomplished in several stages. Throughout, $C$ will denote a generic positive constant, not necessarily the same at each occurrence, which is independent of $\epsilon$.

LEMma 1.

$$
\begin{aligned}
& \left\|\rho^{\epsilon}\right\|_{L^{\infty}\left(J ; H^{1}\left(\Omega_{f}^{\epsilon}\right)\right)}+\left\|\rho_{t}^{\epsilon}\right\|_{L^{2}\left(J ; L^{2}\left(\Omega_{f}^{\epsilon}\right)\right)} \leq C, \\
& \left\|\sigma^{\epsilon}\right\|_{L^{\infty}\left(J ; L^{2}\left(\Omega_{m}^{\epsilon}\right)\right)}+\left\|\sigma_{t}^{\epsilon}\right\|_{L^{2}\left(J ; L^{2}\left(\Omega_{m}^{\epsilon}\right)\right)} \leq C, \\
& \left\|\nabla \sigma^{\epsilon}\right\|_{L^{\infty}\left(J ; L^{2}\left(\Omega_{m}^{\epsilon}\right)\right)} \leq C \epsilon^{-1} .
\end{aligned}
$$

Proof. These are the standard parabolic energy estimates for (4.2); that is, the estimates given by first taking $\varphi=\theta^{\epsilon}$ and then taking $\varphi=\theta_{t}^{\epsilon}$ (which must of course be done on a smooth dense subspace so that the computations can be performed). Note that the domain $\Omega$ is fixed, so that $C$ is indeed independent of $\epsilon$. 
The main properties of the dilation operator that we will need are given by Lemma 2.

LEMMA 2. If $\psi \in L^{2}(\Omega)$ and $\varphi \in L^{2}(\Omega)$ (and $r$ is $m$, $f$, or blank), then

$$
\begin{aligned}
(\tilde{\psi}, \tilde{\varphi})_{\Omega \times \mathcal{Q}_{r}} & =|\mathcal{Q}|(\psi, \varphi)_{\Omega_{r}^{\epsilon}}, \\
\nabla_{y} \tilde{\psi} & =\epsilon \widetilde{\nabla \psi} \\
\|\tilde{\psi}\|_{L^{2}\left(\Omega \times \mathcal{Q}_{r}\right)} & =|\mathcal{Q}|^{1 / 2}\|\psi\|_{L^{2}\left(\Omega_{r}^{\epsilon}\right)}, \\
\left\|\nabla_{y} \tilde{\psi}\right\|_{L^{2}\left(\Omega \times \mathcal{Q}_{r}\right)} & =\epsilon|\mathcal{Q}|^{1 / 2}\|\nabla \psi\|_{L^{2}\left(\Omega_{r}^{\epsilon}\right)}, \\
(\tilde{\psi}, \varphi)_{\Omega \times \mathcal{Q}} & =(\psi, \tilde{\varphi})_{\Omega \times \mathcal{Q}} .
\end{aligned}
$$

Moreover, if $\psi$ is considered to be an element of $L^{2}\left(\Omega \times \mathcal{Q}_{r}\right)$ that is constant in $y$, then $\tilde{\psi} \rightarrow \psi$ as $\epsilon \rightarrow 0$ in $L^{2}\left(\Omega \times \mathcal{Q}_{r}\right)$ strongly.

Proof. The first two results are simple computations:

$$
\begin{aligned}
(\tilde{\psi}, \tilde{\varphi})_{\Omega \times \mathcal{Q}_{r}} & =\int_{\Omega} \int_{\mathcal{Q}_{r}} \psi\left(\epsilon y+c^{\epsilon}(x)\right) \varphi\left(\epsilon y+c^{\epsilon}(x)\right) d y d x \\
& =\int_{\Omega} \int_{\epsilon \mathcal{Q}_{r}+c^{\epsilon}(x)} \psi(z) \varphi(z) \epsilon^{-3} d z d x=|\mathcal{Q}|(\psi, \varphi)_{\Omega_{r}^{\epsilon}} \\
\nabla_{y} \tilde{\psi}(x, y) & =\epsilon \nabla \psi\left(\epsilon y+c^{\epsilon}(x)\right)=\epsilon \widetilde{\nabla \psi}(x, y)
\end{aligned}
$$

The next two results follow from the first two. The fifth result is another computation:

$$
\begin{aligned}
(\tilde{\psi}, \varphi)_{\Omega \times \mathcal{Q}} & =\int_{\Omega} \int_{\mathcal{Q}} \psi\left(\epsilon y+c^{\epsilon}(x)\right) \varphi(x) d y d x \\
& =\int_{\Omega} \epsilon^{-3} \int_{\epsilon \mathcal{Q}+c^{\epsilon}(x)} \psi(z) \varphi(x) d z d x \\
& =\int_{\Omega} \psi(z)\left[\epsilon^{-3} \int_{\epsilon \mathcal{Q}+c^{\epsilon}(z)} \varphi(x) d x\right] d z \\
& =\int_{\Omega} \psi(z) \int_{\mathcal{Q}} \varphi\left(\epsilon y+c^{\epsilon}(z)\right) d y d z=(\psi, \tilde{\varphi})_{\Omega \times \mathcal{Q}}
\end{aligned}
$$

The strong convergence result is clear from the Dominated Convergence Theorem whenever $\psi \in C_{0}^{\infty}(\Omega)$, as then $\psi$ is continuous and bounded on $\Omega$ and $\lim _{\epsilon \rightarrow 0}(\epsilon y+$ $\left.c^{\epsilon}(x)\right)=x$. Since these functions are dense in $L^{2}(\Omega)$, the result follows from the equivalence of norms.

Remark 1. A change of variables shows that the last statement of the lemma is an integral form of Lebesgue's theorem on the differentiation of the integral.

Corollary 1.

$$
\begin{aligned}
& \left\|\tilde{\sigma}^{\epsilon}\right\|_{L^{2}\left(\Omega ; H^{1}\left(\mathcal{Q}_{m} \times J\right)\right)} \leq C, \\
& \left\|\tilde{\rho}^{\epsilon}\right\|_{L^{2}\left(\Omega ; H^{1}\left(J ; L^{2}\left(\mathcal{Q}_{f}\right)\right)\right)} \leq C, \\
& \left\|\nabla_{y} \tilde{\rho}^{\epsilon}\right\|_{L^{2}\left(\Omega ; L^{2}\left(J ; L^{2}\left(\mathcal{Q}_{f}\right)\right)\right)} \leq C \epsilon .
\end{aligned}
$$

Now by Lemma 1 and Corollary 1, we can extract the following weak limits (for 
a subsequence of the $\epsilon^{\prime}$ s):

$$
\begin{aligned}
& \chi_{f}^{\epsilon} \Phi^{*} \rho^{\epsilon} \longrightarrow \Phi \rho \text { in } H^{1}\left(J ; L^{2}(\Omega)\right) \text { weakly, } \\
& \chi_{f}^{\epsilon} \Lambda\left[\nabla \rho^{\epsilon}+\Gamma\left(2 \rho^{\epsilon}-\rho_{\mathrm{ref}}\right)\right] \xi \text { in } L^{2}\left(J ; L^{2}(\Omega)\right) \text { weakly, } \\
& \tilde{\sigma}^{\epsilon} \longrightarrow \sigma \text { in } L^{2}\left(\Omega ; H^{1}\left(\mathcal{Q}_{m} \times J\right)\right) \text { weakly, } \\
& \tilde{\rho}^{\epsilon} \longrightarrow \tau \text { in } L^{2}\left(\Omega ; H^{1}\left(\mathcal{Q}_{f} \times J\right)\right) \text { weakly, }
\end{aligned}
$$

where $\tau=\tau(x, t)$ only, since $\mathcal{Q}_{f}$ is connected. In fact, we claim that $\tau=\rho$. Any $\varphi \in C_{0}^{\infty}(\Omega \times J)$ is also in $L^{2}\left(\Omega ; L^{2}\left(J ; L^{2}\left(\mathcal{Q}_{f}\right)\right)\right)$, so

$$
\left\langle\tilde{\rho}^{\epsilon}, \varphi\right\rangle_{\Omega \times \mathcal{Q}_{f}} \longrightarrow\langle\tau, \varphi\rangle_{\Omega \times \mathcal{Q}_{f}}=\left|\mathcal{Q}_{f}\right|\langle\tau, \varphi\rangle_{\Omega} .
$$

Now Lemma 2 shows that the left-hand side is

$$
\left\langle\tilde{\rho}^{\epsilon}, \varphi\right\rangle_{\Omega \times \mathcal{Q}_{f}}=\left\langle\widetilde{\chi_{f}^{\epsilon} \rho^{\epsilon}}, \varphi\right\rangle_{\Omega \times \mathcal{Q}}=\left\langle\chi_{f}^{\epsilon} \rho^{\epsilon}, \tilde{\varphi}\right\rangle_{\Omega \times \mathcal{Q}} ;
$$

furthermore,

$$
\left\langle\tilde{\rho}^{\epsilon}, \varphi\right\rangle_{\Omega \times \mathcal{Q}_{f}}=\left\langle\chi_{f}^{\epsilon} \rho^{\epsilon}, \tilde{\varphi}\right\rangle_{\Omega \times \mathcal{Q}} \longrightarrow|\mathcal{Q}|\left\langle\left(\Phi^{*}\right)^{-1} \Phi \rho, \varphi\right\rangle_{\Omega}=\left|\mathcal{Q}_{f}\right|\langle\rho, \varphi\rangle_{\Omega},
$$

so that $\tau=\rho$.

We will now find an equation satisfied by $\sigma$. This can be done easily using Lemma 2. Take any $\psi \in L^{2}\left(\Omega ; L^{2}\left(J ; H_{0}^{1}\left(\mathcal{Q}_{m}\right)\right)\right)$. Let

$$
\hat{\psi}(x, z, t)=\left\{\begin{array}{l}
\psi\left(x, \frac{z-c^{\epsilon}(x)}{\epsilon}, t\right) \quad \text { for } z \in \epsilon \mathcal{Q}_{m}+c^{\epsilon}(x), \\
0 \text { for } z \notin \epsilon \mathcal{Q}_{m}+c^{\epsilon}(x) .
\end{array}\right.
$$

Now for almost every fixed $x \in \Omega$, replace the test function in $(4.3)$ by $\hat{\psi}(x, \cdot, t)$ to see that

$$
\begin{aligned}
& \int_{\epsilon \mathcal{Q}_{m}+c^{\epsilon}(x)}\left\{\phi^{\epsilon}(z) \sigma_{t}^{\epsilon}(z, t) \hat{\psi}(x, z, t)\right. \\
& \left.\quad+\epsilon \lambda^{\epsilon}(z)\left[\epsilon \nabla \sigma^{\epsilon}(z, t)+\gamma^{\epsilon}(z)\left(2 \sigma^{\epsilon}(z, t)-\sigma_{\text {ref }}^{\epsilon}(z)\right)\right] \cdot \nabla_{z} \hat{\psi}(x, z, t)\right\} d z \\
& =\int_{\epsilon \mathcal{Q}_{m}+c^{\epsilon}(x)} f(z, t) \hat{\psi}(x, z, t) d z .
\end{aligned}
$$

Upon dilation (i.e., $\left.z \mapsto \epsilon y+c^{\epsilon}(x)\right)$ and integration in $x$ and $t$, we obtain

$$
\left\langle\phi \tilde{\sigma}_{t}^{\epsilon}, \psi\right\rangle_{\Omega \times \mathcal{Q}_{m}}+\left\langle\lambda\left[\nabla_{y} \tilde{\sigma}^{\epsilon}+\gamma\left(2 \tilde{\sigma}^{\epsilon}-\sigma_{\mathrm{ref}}\right)\right], \nabla_{y} \psi\right\rangle_{\Omega \times \mathcal{Q}_{m}}=\langle\tilde{f}, \psi\rangle_{\Omega \times \mathcal{Q}_{m}},
$$

where $\phi, \lambda, \gamma$, and $\sigma_{\text {ref }}$ are integrated over $\mathcal{Q}_{m}$ by their periodicity. As $\epsilon \rightarrow 0$, we see that

$$
\left\langle\phi \sigma_{t}, \psi\right\rangle_{\Omega \times \mathcal{Q}_{m}}+\left\langle\lambda\left[\nabla_{y} \sigma+\gamma\left(2 \sigma-\sigma_{\mathrm{ref}}\right)\right], \nabla_{y} \psi\right\rangle_{\Omega \times \mathcal{Q}_{m}}=\langle f, \psi\rangle_{\Omega \times \mathcal{Q}_{m}} .
$$

This is a weak form of (3.8).

An equation for $\rho$ and $\xi$ can also be derived easily. In (4.2) choose a test function $\varphi \in L^{2}\left(J ; H^{1}(\Omega)\right)$ and then integrate in time. The result is that

$$
\begin{aligned}
& \left\langle\Phi^{*} \rho_{t}^{\epsilon}, \varphi\right\rangle_{\Omega_{f}^{\epsilon}}+\left\langle\Lambda\left[\nabla \rho^{\epsilon}+\Gamma\left(2 \rho^{\epsilon}-\rho_{\mathrm{ref}}\right)\right], \nabla \varphi\right\rangle_{\Omega_{f}^{\epsilon}} \\
& \quad+\left\langle\phi^{\epsilon} \sigma_{t}^{\epsilon}, \varphi\right\rangle_{\Omega_{m}^{\epsilon}}+\left\langle\epsilon \lambda^{\epsilon}\left[\epsilon \nabla \sigma^{\epsilon}+\gamma^{\epsilon}\left(2 \sigma^{\epsilon}-\sigma_{\mathrm{ref}}^{\epsilon}\right)\right], \nabla \varphi\right\rangle_{\Omega_{m}^{\epsilon}}=\langle f, \varphi\rangle_{\Omega} .
\end{aligned}
$$


The first two terms on the left-hand side above tend to $\left\langle\Phi \rho_{t}, \varphi\right\rangle_{\Omega}+\langle\xi, \nabla \varphi\rangle_{\Omega}$, whereas the fourth term tends to zero by Lemma 1 since it has an extra power of $\epsilon$. The third term can be dilated to see its convergence by Lemma 2 :

$$
\left\langle\phi^{\epsilon} \sigma_{t}^{\epsilon}, \varphi\right\rangle_{\Omega_{m}^{\epsilon}}=|\mathcal{Q}|^{-1}\left\langle\phi \tilde{\sigma}_{t}^{\epsilon}, \tilde{\varphi}\right\rangle_{\Omega \times \mathcal{Q}_{m}} \longrightarrow|\mathcal{Q}|^{-1}\left\langle\phi \sigma_{t}, \varphi\right\rangle_{\Omega \times \mathcal{Q}_{m}}=-\left\langle f_{m}, \varphi\right\rangle_{\Omega}
$$

Hence, we have shown that

$$
\left\langle\Phi \rho_{t}, \varphi\right\rangle_{\Omega}+\langle\xi, \nabla \varphi\rangle_{\Omega}=\langle f, \varphi\rangle_{\Omega}+\left\langle f_{m}, \varphi\right\rangle_{\Omega}, \quad \varphi \in L^{2}\left(J ; H^{1}(\Omega)\right) .
$$

Next we will relate $\xi$ to $\rho$. To this end, let us define $\omega_{j}^{\epsilon} \in H^{1}(\Omega)$ by

$$
\omega_{j}^{\epsilon}(x)=\epsilon \mathcal{E} \omega_{j}\left(\frac{x-c^{\epsilon}(x)}{\epsilon}\right),
$$

where $\mathcal{E}: H^{1}\left(\mathcal{Q}_{f}\right) \rightarrow H^{1}(\mathcal{Q})$ is some bounded extension operator (for example, the one of Calderón [8]). Note that $\tilde{\omega}_{j}^{\epsilon}(x, y)=\epsilon \mathcal{E} \omega_{j}(y)$ and $\widetilde{\nabla \omega_{j}^{\epsilon}}(x, y)=\nabla \mathcal{E} \omega_{j}(y)$. Also let

$$
w_{i j}=\frac{1}{|\mathcal{Q}|} \int_{\mathcal{Q}_{f}} \partial_{i} \omega_{j}(y) d y
$$

LEMma 3.

$$
\begin{aligned}
& \omega_{j}^{\epsilon} \longrightarrow 0 \quad \text { in } L^{2}(\Omega) \text { strongly } \\
& \epsilon \nabla \omega_{j}^{\epsilon} \longrightarrow 0 \quad \text { in } L^{2}(\Omega) \text { strongly } \\
& \chi_{f}^{\epsilon} \partial_{i} \omega_{j}^{\epsilon} \longrightarrow w_{i j} \quad \text { in } L^{2}(\Omega) \text { weakly. }
\end{aligned}
$$

Proof. For the first limit, note that

$$
\left\|\omega_{j}^{\epsilon}\right\|_{L^{2}(\Omega)}=|\mathcal{Q}|^{-1 / 2}\left\|\tilde{\omega}_{j}^{\epsilon}\right\|_{L^{2}(\Omega \times \mathcal{Q})}=|\mathcal{Q}|^{-1 / 2}|\Omega| \epsilon\left\|\mathcal{E} \omega_{j}\right\|_{L^{2}(\mathcal{Q})} \leq C \epsilon\left\|\omega_{j}\right\|_{L^{2}\left(\mathcal{Q}_{f}\right)} \longrightarrow 0 .
$$

The second limit is similar, noting that

$$
\left\|\nabla \omega_{j}^{\epsilon}\right\|_{L^{2}(\Omega)}=|\mathcal{Q}|^{-1 / 2}\left\|\widetilde{\nabla \omega_{j}^{\epsilon}}\right\|_{L^{2}(\Omega \times \mathcal{Q})}=|\mathcal{Q}|^{-1 / 2}|\Omega|\left\|\nabla \mathcal{E} \omega_{j}\right\|_{L^{2}(\mathcal{Q})} \leq C\left\|\nabla \omega_{j}\right\|_{L^{2}\left(\mathcal{Q}_{f}\right)} .
$$

The above expression bounds the $L^{2}(\Omega)$-norm of $\chi_{f}^{\epsilon} \partial_{i} \omega_{j}^{\epsilon}$, so this expression has some weak limit. To see what this limit is, solve the elliptic problem modulo constants

$$
\begin{aligned}
& -\Delta \psi=\chi_{\mathcal{Q}_{f}} \partial_{i} \omega_{j}-w_{i j} \quad \text { for } y \in \mathcal{Q}, \\
& \nabla \psi \cdot \nu=0 \quad \text { for } y \in \partial \mathcal{Q}
\end{aligned}
$$

where $\chi_{\mathcal{Q}_{f}}$ is the characteristic function of $\mathcal{Q}_{f}$. We can do this since the average of $\chi_{\mathcal{Q}_{f}} \partial_{i} \omega_{j}-w_{i j}$ is zero. Note that $\psi \in H^{1}(\mathcal{Q})$. Now for $\varphi \in C_{0}^{\infty}$,

$$
\begin{aligned}
\left(\chi_{f}^{\epsilon} \partial_{i} \omega_{j}^{\epsilon}-w_{i j}, \varphi\right)_{\Omega} & =|\mathcal{Q}|^{-1}\left(\tilde{\chi}_{f}^{\epsilon} \widetilde{\partial_{i} \omega_{j}^{\epsilon}}-w_{i j}, \tilde{\varphi}\right)_{\Omega \times \mathcal{Q}}=|\mathcal{Q}|^{-1}\left(\chi_{\mathcal{Q}_{f}} \partial_{i} \omega_{j}-w_{i j}, \tilde{\varphi}\right)_{\Omega \times \mathcal{Q}} \\
& =|\mathcal{Q}|^{-1}(-\Delta \psi, \tilde{\varphi})_{\Omega \times \mathcal{Q}}=|\mathcal{Q}|^{-1}(\nabla \psi, \epsilon \widetilde{\nabla \varphi})_{\Omega \times \mathcal{Q}} \leq C \epsilon \longrightarrow 0 .
\end{aligned}
$$

From the definition (3.2)-(3.3) of $\omega_{j}$ we can see that

$$
0=-\left(\nabla \cdot\left(\nabla \omega_{j}^{\epsilon}+e_{j}\right), \psi\right)_{\Omega_{f}^{\epsilon}}=\left(\nabla \omega_{j}^{\epsilon}+e_{j}, \nabla \psi\right)_{\Omega_{f}^{\epsilon}}, \quad \psi \in H_{0}^{1}(\Omega) .
$$


For any $\varphi \in C_{0}^{\infty}$, take $\psi=\rho^{\epsilon} \Lambda \varphi$ above. After adding and subtracting the same term twice and after integrating in time, we have that

$$
\begin{aligned}
& \left\langle\nabla \omega_{j}^{\epsilon}, \rho^{\epsilon} \nabla(\Lambda \varphi)-\varphi \Lambda \Gamma\left(2 \rho^{\epsilon}-\rho_{\mathrm{ref}}\right)\right\rangle_{\Omega_{f}^{\epsilon}}+\left\langle e_{j}, \rho^{\epsilon} \nabla(\Lambda \varphi)-\varphi \Lambda \Gamma\left(2 \rho^{\epsilon}-\rho_{\mathrm{ref}}\right)\right\rangle_{\Omega_{f}^{\epsilon}} \\
& \quad+\left\langle\nabla \omega_{j}^{\epsilon}, \varphi \Lambda\left[\nabla \rho^{\epsilon}+\Gamma\left(2 \rho^{\epsilon}-\rho_{\mathrm{ref}}\right)\right]\right\rangle_{\Omega_{f}^{\epsilon}}+\left\langle e_{j}, \varphi \Lambda\left[\nabla \rho^{\epsilon}+\Gamma\left(2 \rho^{\epsilon}-\rho_{\mathrm{ref}}\right)\right]\right\rangle_{\Omega_{f}^{\epsilon}}=0 .
\end{aligned}
$$

The first term converges by dilation:

$$
\begin{aligned}
\left\langle\nabla \omega_{j}^{\epsilon},\right. & \left.\rho^{\epsilon} \nabla(\Lambda \varphi)-\varphi \Lambda \Gamma\left(2 \rho^{\epsilon}-\rho_{\mathrm{ref}}\right)\right\rangle_{\Omega_{f}^{\epsilon}} \\
& =|\mathcal{Q}|^{-1}\left\langle\nabla \omega_{j}, \tilde{\rho}^{\epsilon} \widetilde{\nabla(\Lambda \varphi)}-\widetilde{\varphi \Lambda \Gamma}\left(2 \tilde{\rho}^{\epsilon}-\tilde{\rho}_{\mathrm{ref}}\right)\right\rangle_{\Omega \times \mathcal{Q}_{f}} \\
& \longrightarrow|\mathcal{Q}|^{-1}\left\langle\nabla \omega_{j}, \rho \nabla(\Lambda \varphi)-\varphi \Lambda \Gamma\left(2 \rho-\rho_{\mathrm{ref}}\right)\right\rangle_{\Omega \times \mathcal{Q}_{f}} \\
& =\sum_{i=1}^{3}\left\langle w_{i j}, \rho \partial_{i}(\Lambda \varphi)-\varphi \Lambda \Gamma_{i}\left(2 \rho-\rho_{\mathrm{ref}}\right)\right\rangle_{\Omega} .
\end{aligned}
$$

The second term converges trivially to

$$
\frac{\left|\mathcal{Q}_{f}\right|}{|\mathcal{Q}|}\left\langle e_{j}, \rho \nabla(\Lambda \varphi)-\varphi \Lambda \Gamma\left(2 \rho-\rho_{\mathrm{ref}}\right)\right\rangle_{\Omega}
$$

since $\chi_{f}^{\epsilon} \rightarrow\left|\mathcal{Q}_{f}\right| /|\mathcal{Q}|$ in $L^{2}(\Omega)$ weakly is easily shown from Lemma 2 . The convergence of the third term is found from (4.2) by taking the test function $\omega_{j}^{\epsilon} \varphi$. Integration in time shows that

$$
\begin{aligned}
& \left\langle\Phi^{*} \rho_{t}^{\epsilon}, \omega_{j}^{\epsilon} \varphi\right\rangle_{\Omega_{f}^{\epsilon}}+\left\langle\Lambda\left[\nabla \rho^{\epsilon}+\Gamma\left(2 \rho^{\epsilon}-\rho_{\mathrm{ref}}\right)\right], \omega_{j}^{\epsilon} \nabla \varphi+\varphi \nabla \omega_{j}^{\epsilon}\right\rangle_{\Omega_{f}^{\epsilon}} \\
& \quad+\left\langle\phi^{\epsilon} \sigma_{t}^{\epsilon}, \omega_{j}^{\epsilon} \varphi\right\rangle_{\Omega_{m}^{\epsilon}}+\left\langle\epsilon \lambda^{\epsilon}\left[\epsilon \nabla \sigma^{\epsilon}+\gamma^{\epsilon}\left(2 \sigma^{\epsilon}-\sigma_{\mathrm{ref}}^{\epsilon}\right)\right], \omega_{j}^{\epsilon} \nabla \varphi+\varphi \nabla \omega_{j}^{\epsilon}\right\rangle_{\Omega_{m}^{\epsilon}}=\left\langle f, \omega_{j}^{\epsilon} \varphi\right\rangle_{\Omega} .
\end{aligned}
$$

Lemmas 1 and 3 now show that

$$
\lim _{\epsilon \rightarrow 0}\left\langle\nabla \omega_{j}^{\epsilon}, \varphi \Lambda\left[\nabla \rho^{\epsilon}+\Gamma\left(2 \rho^{\epsilon}-\rho_{\mathrm{ref}}\right)\right]\right\rangle_{\Omega_{f}^{\epsilon}}=0 .
$$

Hence we conclude that the fourth term of (4.7) converges to

$$
\begin{aligned}
& \left\langle\xi_{j}, \varphi\right\rangle_{\Omega} \\
& =\sum_{i=1}^{3}\left\{-\left\langle\left[\frac{\left|\mathcal{Q}_{f}\right|}{|\mathcal{Q}|} \delta_{i j}+w_{i j}\right] \rho, \partial_{i}(\Lambda \varphi)\right\rangle_{\Omega}+\left\langle\left[\frac{\left|\mathcal{Q}_{f}\right|}{|\mathcal{Q}|} \delta_{i j}+w_{i j}\right] \Lambda \Gamma_{i}\left(2 \rho-\rho_{\mathrm{ref}}\right), \varphi\right\rangle_{\Omega}\right\}
\end{aligned}
$$

which is the distributional form of

$$
\xi=\frac{K}{\mu c}\left[\nabla \rho+\Gamma\left(2 \rho-\rho_{\mathrm{ref}}\right)\right] .
$$

Hence (4.5) is a weak form of (3.5)-(3.6), (3.11).

The following trivial proposition enables us to derive the boundary and initial conditions satisfied by our limit functions.

Proposition. If $\mathcal{T}: X \rightarrow Y$ is a continuous linear operator between Banach spaces $X$ and $Y$, and if $\psi^{\epsilon} \rightarrow \psi$ in $X$ weakly, then $\mathcal{T} \psi^{\epsilon} \rightarrow \mathcal{T} \psi$ in $Y$ weakly.

If $\mathcal{T}_{0}$ is the linear operator giving the trace for time zero, then [15] $\mathcal{T}_{0}: H^{1}(\Omega \times$ $J) \rightarrow H^{1 / 2}(\Omega)$ is bounded. Hence

$$
\mathcal{T}_{0}\left(\chi_{f}^{\epsilon} \Phi^{*} \rho^{\epsilon}\right) \longrightarrow \mathcal{T}_{0}(\Phi \rho)=\Phi \mathcal{T}_{0} \rho
$$


But

$$
\mathcal{T}_{0}\left(\chi_{f}^{\epsilon} \Phi^{*} \rho^{\epsilon}\right)=\chi_{f}^{\epsilon} \Phi^{*} \rho_{\text {init }} \longrightarrow \Phi \rho_{\text {init }}
$$

consequently, we have (3.7). For almost every $x$, we also have (3.10) since, for the appropriate trace operator,

$$
\mathcal{T}_{0} \tilde{\sigma}^{\epsilon}(x, y) \longrightarrow \mathcal{T}_{0} \sigma(x, y)
$$

and

$$
\mathcal{T}_{0} \tilde{\sigma}^{\epsilon}(x, y)=\tilde{\rho}_{\text {init }}(x, y) \longrightarrow \rho_{\text {init }}(x) .
$$

If now $\mathcal{T}_{b}: H^{1}\left(\mathcal{Q}_{r} \times J\right) \rightarrow H^{1 / 2}\left(\partial \mathcal{Q}_{r} \times J\right), r=m$ or $f$, is the boundary trace operator, then for almost every $x$

$$
\mathcal{T}_{b} \tilde{\sigma}^{\epsilon}(x, y, t) \longrightarrow \mathcal{T}_{b} \sigma(x, y, t)
$$

and

$$
\mathcal{T}_{b} \tilde{\sigma}^{\epsilon}(x, y, t)=\mathcal{T}_{b} \tilde{\rho}^{\epsilon}(x, y, t) \longrightarrow \mathcal{T}_{b} \rho(x, t)=\rho(x, t) .
$$

This shows (3.9).

We now have a solution to the macroscopic model. To see that this solution is unique, assume that $\rho$ and $\sigma$ are the differences of two solutions. These then satisfy the macroscopic model's equations with $f \equiv \rho_{\text {init }} \equiv 0$ and the gravitational pseudosource terms $\nabla \cdot\left((K / \mu) g \rho_{\text {ref }}^{2}\right)$ and $\nabla \cdot\left((k / \mu) g \sigma_{\text {ref }}^{2}\right)$ set to zero. Multiply (3.5) by $\rho$ and integrate in $x$ to see that

$$
\left(\Phi \rho_{t}, \rho\right)_{\Omega}+\left(\frac{K}{\mu c}\left[\nabla \rho+2 c g \rho \rho_{\mathrm{ref}}\right], \nabla \rho\right)_{\Omega}=\left(f_{m}, \rho\right)_{\Omega} .
$$

Now multiply (3.8) by $\sigma-\rho$ and integrate in both $x$ and $y$ to obtain

$$
\left(\phi \sigma_{t}, \sigma\right)_{\Omega \times \mathcal{Q}_{m}}-\left(\phi \sigma_{t}, \rho\right)_{\Omega \times \mathcal{Q}_{m}}+\left(\frac{k}{\mu c}\left[\nabla_{y} \sigma+2 c g \sigma \sigma_{\mathrm{ref}}\right], \nabla_{y} \sigma\right)_{\Omega \times \mathcal{Q}_{m}}=0
$$

since $\nabla_{y} \rho=0$. Note that $f_{m}=-|\mathcal{Q}|^{-1}\left(\phi \sigma_{t}, 1\right)_{\mathcal{Q}_{m}}$ cancels when $|\mathcal{Q}|$ times the first equation is added to the second. Hence standard energy estimates of this combined equation show the uniqueness. Finally, since the solution is unique, the entire sequence of solutions to the microscopic model converges as required, and the proof of Theorem 2 is complete.

Of course, our macroscopic coefficients should have the appropriate properties. Obviously $\Phi$ is uniformly positive. As for $K$, we have Theorem 3 .

THEOREM 3. The macroscopic fracture permeability tensor $K(x)$ is symmetric and positivedefinite.

Proof. From (3.2)-(3.3), we see that

$$
0=-\left(\nabla \cdot\left(\nabla \omega_{j}+e_{j}\right), \omega_{i}\right)_{\mathcal{Q}_{f}}=\left(\nabla \omega_{j}+e_{j}, \nabla \omega_{i}\right)_{\mathcal{Q}_{f}}
$$

by the periodicity of the $\omega_{k}$. Hence

$$
\left(\partial_{j} \omega_{i}, 1\right)_{\mathcal{Q}_{f}}=-\left(\nabla \omega_{j}, \nabla \omega_{i}\right)_{\mathcal{Q}_{f}}
$$


which shows that $K$ is symmetric. In fact, we can use this to rewrite $K_{i j}$ :

$$
\begin{aligned}
|\mathcal{Q}|\left(K^{*}\right)^{-1} K_{i j} & =\left|\mathcal{Q}_{f}\right| \delta_{i j}+\left(\partial_{i} \omega_{j}, 1\right)_{\mathcal{Q}_{f}} \\
& =\left(\nabla y_{j}, \nabla y_{i}\right)_{\mathcal{Q}_{f}}+\left(\partial_{i} \omega_{j}, 1\right)_{\mathcal{Q}_{f}}+\left(\partial_{j} \omega_{i}, 1\right)_{\mathcal{Q}_{f}}-\left(\partial_{j} \omega_{i}, 1\right)_{\mathcal{Q}_{f}} \\
& =\left(\nabla y_{j}, \nabla y_{i}\right)_{\mathcal{Q}_{f}}+\left(\nabla \omega_{j}, \nabla y_{i}\right)_{\mathcal{Q}_{f}}+\left(\nabla \omega_{i}, \nabla y_{j}\right)_{\mathcal{Q}_{f}}+\left(\nabla \omega_{j}, \nabla \omega_{i}\right)_{\mathcal{Q}_{f}} \\
& =\left(\nabla\left(y_{j}+\omega_{j}\right), \nabla\left(y_{i}+\omega_{i}\right)\right)_{\mathcal{Q}_{f}}
\end{aligned}
$$

This shows that $K$ is positive semidefinite. Definiteness follows from the connectedness of $\mathcal{Q}_{f}$ and the periodicity of the $\omega_{j}$. Let $\xi$ be any constant vector. Then, with $\omega$ being the vector whose components are the $\omega_{j}$,

$$
0=\sum_{i, j} \xi_{j}\left(\nabla\left(y_{j}+\omega_{j}\right), \nabla\left(y_{i}+\omega_{i}\right)\right)_{\mathcal{Q}_{f}} \xi_{i}=\sum_{k}\left(\partial_{k}[\xi \cdot(y+\omega)], \partial_{k}[\xi \cdot(y+\omega)]\right)_{\mathcal{Q}_{f}} .
$$

Since $\mathcal{Q}_{f}$ is connected, $\omega(y) \cdot \xi=\alpha-y \cdot \xi$ for some constant $\alpha$. This being periodic in $y$ forces $\xi$ to be zero.

Remark 2 . The tensor $K$ may not be strictly positive definite for some degenerate (disconnected) geometries. For example, in one space dimension $\mathcal{Q}_{f}$ consists of two disjoint intervals, so $K \equiv 0$.

We close by noting that the macroscopic model is well posed.

THEOREM 4. If $\partial \Omega$ is smooth, then $\rho \in H^{1}\left(J ; L^{2}(\Omega)\right) \cap L^{2}\left(J ; H^{2}(\Omega)\right)$ and $\sigma \in L^{2}\left(\Omega ; H^{1}\left(J ; L^{2}\left(\mathcal{Q}_{m}\right)\right)\right) \cap L^{2}\left(\Omega ; L^{2}\left(J ; H^{2}\left(\mathcal{Q}_{m}\right)\right)\right)$. Moreover, the solution varies continuously with the data $f \in L^{2}\left(J ; L^{2}(\Omega)\right), \rho_{\text {init }} \in H^{1}(\Omega), \rho_{\text {ref }} \in W^{1, \infty}(\Omega)$, and $\sigma_{\text {ref }} \in W^{1, \infty}\left(\mathcal{Q}_{m}\right)$.

Proof. The theorem was proven by Arbogast for the original formulation of the model using the method of continuity [3]. He also proved it for a generalization of the model that included gravity in the matrix (and had a more complicated definition of the matrix source term in that the fracture density was assumed to vary linearly in space over each block) [2]. This latter proof is the most convenient for the present situation. It treats the matrix source term in much the same way as we did in proving uniqueness of the solution; that is, it drops out of the equations after properly combining (3.5) and (3.8). The external source in the matrix equations can be dealt with by combining it with the gravitational pseudo-source term. By properly taking into account the subtle differences between the homogenized and original formulations of the model mentioned at the end of $\S 3$ (and by noting that the fracture flow is simply constant in space over each block), the proof goes through easily.

\section{REFERENCES}

[1] B. Amaziane And A. Bourgeat, Effective behavior of two-phase flow in heterogeneous reservoir, in Numerical Simulation in Oil Recovery, M. F. Wheeler, ed., the IMA Volumes in Mathematics and its Applications 11, Springer-Verlag, Berlin, New York, 1988, pp. 1-22.

[2] T. Arbogast, The double porosity model for single phase flow in naturally fractured reservoirs, in Numerical Simulation in Oil Recovery, M. F. Wheeler, ed., the IMA Volumes in Mathematics and its Applications 11, Springer-Verlag, Berlin, New York, 1988, pp. 23-45.

[3] T. Arbogast, Analysis of the simulation of single phase flow through a naturally fractured reservoir, SIAM J. Numer. Anal., 26 (1989), pp. 12-29.

[4] T. Arbogast, J. Douglas, JR., And U. Hornung, Modeling of naturally fractured reservoirs by formal homogenization techniques (to appear). 
[5] G. I. Barenblatt, Iu. P. Zheltov, and I. N. Kochina, Basic concepts in the theory of seepage of homogeneous liquids in fissured rocks [strata], Prikl. Mat. Mekh., 24 (1960), pp. 852-864; J. Appl. Math. Mech., 24 (1960), pp. 1286-1303.

[6] A. Bensoussan, J. L. Lions, and G. Papanicolaou, Asymptotic Analysis for Periodic Structures, North-Holland, Amsterdam, 1978.

[7] A. Bourgeat, Homogenization of two phase flow equations, Proc. Sympos. Pure Math., 45 (1986), pp. 157-163.

[8] A. P. Calderón, Lebesgue spaces of differentiable functions and distributions, Proc. Sympos. Pure Math., 4 (1961), pp. 33-49.

[9] J. Douglas, JR., AND T. Arbogast, Dual-porosity models for flow in naturally fractured reservoirs, in Dynamics of Fluids in Hierarchical Porous Media, J. H. Cushman, ed., Academic Press, London, 1990, pp. 177-221.

[10] J. Douglas, Jr., P. J. Paes Leme, T. Arbogast, and T. Schmitt, Simulation of flow in naturally fractured reservoirs, Paper SPE 16019, in Proc., 9th SPE Symposium on Reservoir Simulation, Society of Petroleum Engineers, Dallas, TX, 1987, pp. 271-279.

[11] U. Hornung, Miscible displacement in porous media influenced by mobile and immobile water, Tech. Report \#102,, Department of Mathematics, Arizona State University, Tempe, AZ (1987).

[12] U. Hornung AND W. JÄGER, A model for chemical reactions in porous media, in Complex Chemical Reaction Systems. Mathematical Modelling and Simulation, J. Warnatz and W. Jäger, eds., Springer Series in Chemical Physics 47, Springer-Verlag, Berlin, New York, 1987, pp. 318-334.

[13] U. Hornung and R. Showalter, Diffusion models for fractured media, J. Math. Anal. Appl., to appear.

[14] H. Kazemi, Pressure transient analysis of naturally fractured reservoirs with uniform fracture distribution, Soc. Petroleum Engrs. J., 9 (1969), pp. 451-462.

[15] J. L. Lions and E. Magenes, Non-Homogeneous Boundary Value Problems and Applcations 1, Springer-Verlag, Berlin, New York, 1970.

[16] S. J. Pirson, Performance of fractured oil reservoirs, Bull. Amer. Assoc. Petroleum Geologists, 37 (1953), pp. 232-244.

[17] E. Sanchez-Palencia, Non-homogeneous Media and Vibration Theory, Lecture Notes in Physics 127, Springer-Verlag, Berlin, New York, 1980.

[18] A. DE SwaAn, Analytic solutions for determining naturally fractured reservoir properties by well testing, Soc. Petroleum Engrs. J., 16 (1976), pp. 117-122.

[19] L. TARTAR, Incompressible fluid flow in a porous medium-convergence of the homogenization process, in Non-homogeneous Media and Vibration Theory, E. Sanchez-Palencia, Lecture Notes in Physics 127, Springer-Verlag, Berlin, New York, 1980, pp. 368-377.

[20] Ch. Vogt, A homogenization theorem leading to a Volterra integro-differential equation for permeation chromatography, Preprint \#155, Sonderforschungsbereich 123, Heidelberg, 1982.

[21] J. E. Warren And P. J. Root, The behavior of naturally fractured reservoirs, Soc. Petroleum Engrs. J., 3 (1963), pp. 245-255. 